\title{
Spectrum, Manifestations and Outcomes of Dengue Infection in Individuals with and without Liver Disease
}

\author{
Anand V. Kulkarni ${ }^{1}$, Ashok K. Choudhury ${ }^{1}$, Madhumita Premkumar ${ }^{1}$, Priyanka Jain ${ }^{3}$, \\ Ekta Gupta ${ }^{2}$ and Shiv Kumar Sarin*1 \\ ${ }^{1}$ Department of Hepatology and Transplant, Institute of Liver and Biliary Sciences, New Delhi, India; ${ }^{2}$ Department of Virology, \\ Institute of Liver and Biliary Sciences, New Delhi, India; ${ }^{3}$ Department of Statistics, Institute of Liver and Biliary Sciences, New \\ Delhi, India
}

\begin{abstract}
Background and Aims: Dengue infection is a major health burden, which can result in mild self-limited febrile illness to highly fatal hemorrhagic disease. There is paucity of literature describing the manifestations of dengue in patients with underlying liver disease. We studied and compared the manifestations of this tropical infection in patients with and without liver disease. Methods: Patients with serologically-confirmed dengue infection were included in this retrospective study, obtained for over a period of 1 year. Demographic and laboratory variables were compared for the individuals with no underlying liver disease (Group A, $n=71$ ), chronic hepatitis (Group B, $n=12$ ), and cirrhosis (Group C, $n=12$ ). Results: Males predominated the study population $(61 \%)$, with a higher mean age in the cirrhotic group. The most common clinical manifestation was classical dengue fever, seen in 89 individuals. Two presented as dengue hemorrhagic fever ( 1 each in Group $A$ and Group B), 1 presented as acute liver failure, and 3 as acute-onchronic liver failure. Hemoconcentration was less evident in Group C as compared to Group A and Group B $(p<0.001)$. Patients in Group $C$ had significantly prolonged International Normalized Ratio (INR) and enhanced thrombocytopenia compared to patients in Group A and Group B. Patients in Group C also required prolonged hospital stay (Group A: $4.83 \pm 2.88$, Group B: $7.33 \pm 2.3$, Group C: $13 \pm 5$ days; $p<0.001)$. Three patients expired in Group $C$ compared to the 1 in Group $A$ and none in Group $B(p=0.01)$. On univariate analysis, hemoglobin, albumin, INR, and bilirubin predicted development of liver failure. On multivariate analysis, INR and bilirubin predicted development of liver failure. Conclusions: Dengue infection can have varied manifestations, ranging from simple fever to acute-on-chronic liver failure and acute liver failure. Cirrhotic patients lack classical features of dengue and have relatively poor prognosis. Dengue should be suspected as a cause of liver failure in endemic areas, where no etiological cause is discernible.
\end{abstract}

Keywords: Dengue; Cirrhosis; Acute-on-chronic liver failure; Acute liver failure. Abbreviations: ACLF, acute-on-chronic liver failure; ALF, acute liver failure; ALT, alanine transaminase; AST, aspartate transaminase; $\mathrm{DF}$, dengue fever; $\mathrm{DHF}$, dengue hemorrhagic fever; DSS, dengue shock syndrome; HBV, hepatitis B virus; HCV, hepatitis $C$ virus; INR, International Normalized Ratio; NASH, non-alcoholic steatohepatitis; ULN, upper limit of normal.

Received: 14 August 2018; Revised: 21 January 2019; Accepted: 12 February 2019 *Correspondence to: Shiv Kumar Sarin, Department of Hepatology, Institute of Liver \& Biliary Sciences (ILBS), D-1, Vasant Kunj, New Delhi 110070, India. Tel: +91-11-46300000, Fax: +91-11-46300010, E-mail: shivsarin@gmail.com; sksarin@ilbs.in
Citation of this article: Kulkarni AV, Choudhury AK, Premkumar M, Jain P, Gupta E, Sarin SK. Spectrum, manifestations and outcomes of dengue infection in individuals with and without liver disease. J Clin Transl Hepatol 2019;7(2):106-111. doi: $10.14218 / \mathrm{JCTH} .2018 .00047$.

\section{Introduction}

Dengue virus is a single-stranded, enveloped RNA virus, representing four antigenically distinct serotypes (DENV1, DENV2, DENV3, and DENV4). It is a crucial arboviral disease in terms of geographical distribution, morbidity, and mortality. More than half a million cases of dengue hemorrhagic fever (DHF) occur each year, with at least 12,000 deaths. Mortality ranges between $10 \%$ and $20 \%$, but in dengue shock syndrome (DSS) the mortality rates can reach up to $40 \%$. The changing prevalence shows that approximately 390 million dengue infections occur per year, of which around 96 million manifest clinically. ${ }^{1}$

Dengue virus, transmitted by the Aedes aegypti mosquito, can result in mild self-limited febrile illness to highly fatal hemorrhagic disease. After 5-8 days following the mosquito bite, the disease begins with fever, severe headache, and any of the following: retroorbital pain with or without photophobia; backache; and, myalgia and arthralgia. Dengue can cause multiorgan dysfunction, myocarditis, encephalitis, renal failure, and most importantly hepatitis. According to the latest World Health Organization guidelines, dengue is classified as dengue (with or without warning signs) or severe dengue. ${ }^{2,3}$

Liver involvement in dengue can cause painful hepatomegaly, transaminitis and jaundice in varying levels. ${ }^{4}$ Transaminitis is seen in $65-97 \%$ of patients. ${ }^{5,6}$ Aspartate transaminase (AST) is elevated more than alanine transaminase (ALT), as AST has sources other than liver that are involved in dengue infection, namely the muscle. The elevation in aminotransferases is multifactorial and is attributed to viral factors, immunological factors and anoxia, leading to apoptosis and midzonal necrosis. ${ }^{7}$ Liver biopsy may show features of centrilobular necrosis, acidophil bodies and monocyte infiltration of the portal tract in DHF/ DSS, which is similar to the features noted in acute severe hepatitis. ${ }^{8,9}$ The clinical presentation usually includes incidentally detected acute hepatitis to acute liver failure (ALF). The incidence of ALF due to dengue infection in adults is reported to be between $5.2 \%$ and $8.9 \% .{ }^{10,11}$ However, 
there are hardly any case reports of dengue causing acuteon-chronic liver failure (ACLF). ${ }^{12}$

Liver involvement has been reported to prolong the clinical course of the disease, although it is not often considered a poor prognostic sign in normal individuals. There is a profound deficiency in the knowledge regarding liver involvement, the spectrum of clinical presentation, and the impact of dengue infection in patients with liver disease. The present study was hospital-based and the referred cases of dengue infection with liver involvement irrespective of pre-existing liver disease were admitted and included. The primary objective was to address the clinical presentation, biochemical alteration and outcomes of dengue infection in individuals with chronic hepatitis and cirrhosis in comparison to those having dengue infection in the absence of pre-existing liver disease.

\section{Methods}

\section{Study design}

This single-center, hospital-based retrospective study used data from April 2016 back to July 2015 for patients treated in the Department of Hepatology. All patients who had been admitted with clinical suspicion of dengue fever which was confirmed either by Dengue NS1 antigen positivity and/or serology, irrespective of the pre-existing liver disease (known or unknown) and aged between 18-70 years-old were included. The patients were categorized as DF, DHF or DSS as far as the dengue diagnosis was concerned. For liver involvement, the patients were grouped as asymptomatic, acute hepatitis, ACLF and ALF. Patients with other cause for fever, confirmed sepsis, or pregnancy were excluded.

Patients presenting with fever were evaluated with a detailed history-taking and physical examination, followed by investigations for lower respiratory tract infection, urinary tract infection, malaria, and leptospira. All patients were tested for the malarial parasite by thick smear examination. Chest x-ray was performed, as well as sepsis screening (by urine examination and culture sensitivity, blood culture sensitivity) and the leptospira IgM antibody test, if deemed necessary. In the presence of a seasonal outbreak or an epidemic of dengue fever, the presence of high-grade fever, bone pain and thrombocytopenia or bleeding, led to suspicion of dengue infection. NS1 antigen was tested for those with less than 6 days of fever, and serology was performed for those presenting after 6 days. Confirmed cases were enrolled into the study. Blood was collected from symptomatic patients and diagnosis was confirmed by dengue-specific NS1 ELISA test (Panbio Diagnostics, Australia) in acute phase samples and in patients with more than 6 days of fever; the dengue-specific IgM antibodies capture ELISA (Panbio Diagnostics, Australia) was carried out as per the manufacturer's instruction. Detection of the circulating serotype of the virus was carried out in a few acute phase samples by modified multiplex RT-PCR kits. ${ }^{13}$

Being a tertiary liver hospital, we had patients with known or unknown cases of liver disease, like-chronic hepatitis [related to hepatitis B virus (HBV), hepatitis C virus (HCV), alcoholic and non-alcoholic steatohepatitis (NASH for the latter), etc. ], cirrhosis on follow-up and some being referred in view of deranged LFT. Patients were admitted, evaluated and treated with adequate hydration, cold sponging and antipyretics. Antibiotics were upgraded as per culture reports in patients with superadded sepsis. Hydration was performed judiciously in patients with liver disease, mainly as $5 \%$ albumin or isotonic balanced solution (i.e. Plasma Lyte). Platelet count $\leq 10000 / c c$ and/or presence of hemorrhagic manifestation was the threshold for platelet transfusion. Hemogram was repeated daily and hydration was assessed every $6 \mathrm{~h}$. Enteral nutrition was the preferred route by oral or nasogastric tube, unless contraindicated. Patients were managed in the ward or high-dependency unit; if there was any clinical deterioration or patients developed DHF/DSS, they were shifted to the dedicated liver intensive care unit and were intubated and mechanically ventilated, when indicated. Periodic sepsis screening and organ failure support was continued till recovery or discharge from hospital.

\section{Study definitions}

The standard definitions as per pre-existing guidelines were utilized. ${ }^{14-16}$

Dengue fever was defined as fever with at least two of the following features: ocular pain, headache, muscle or joint pains, cutaneous rash, bleeding and reduced leukocyte count.

DHF was defined as fever, thrombocytopenia ( $\left.\leq 100 \times 10^{9} / \mathrm{L}\right)$, bleeding and evidence of plasma leakage.

DSS was defined as DHF with tachycardia or low pulse pressure $(<20 \mathrm{mmHg}$ ) or hypotension (systolic blood pressure $<90 \mathrm{mmHg}$ ).

Chronic hepatitis/chronic liver disease in absence of cirrhosis included hepatitis due to HBV, HCV, autoimmune response, alcohol or NASH, etc. that had been diagnosed previously and on follow-up or diagnosed during current admission.

Cirrhosis was defined by clinical features of chronic liver disease and/or presence of portal hypertension. Patients with previously diagnosed cirrhosis were included in group $\mathrm{C}$.

Acute hepatitis (Dengue-related) was defined as $\geq 2$ times the upper limit of normal (ULN) of AST /ALT (normal: 0-40 IU/mL).

ACLF was defined as acute hepatic insult manifesting as jaundice and coagulopathy, complicated within 4 weeks by ascites and/or encephalopathy in a patient with previously diagnosed or undiagnosed chronic liver disease.

ALF was defined by jaundice to encephalopathy interval of $<28$ days.

Patients with no underlying liver disease were classified as Group A, while those with underlying chronic hepatitis were grouped into Group B; lastly, patients with underlying cirrhosis were grouped into Group C.

\section{Statistical analysis}

Categorical variables were presented as proportions while continuous variables were either presented as mean (standard deviation) or median (range). Comparison of two continuous variables was done by independent $t$-test or Mann-Whitney test for parametric or non-parametric data, respectively, while categorical variables by Fisher's exact test or Pearson's Chi-square test. Comparison of continuous variables was done by one way ANOVA with Post hoc by Bonferroni method for parametric data or Kruskal-Wallis test for non-parametric data. The predictors of liver failure 


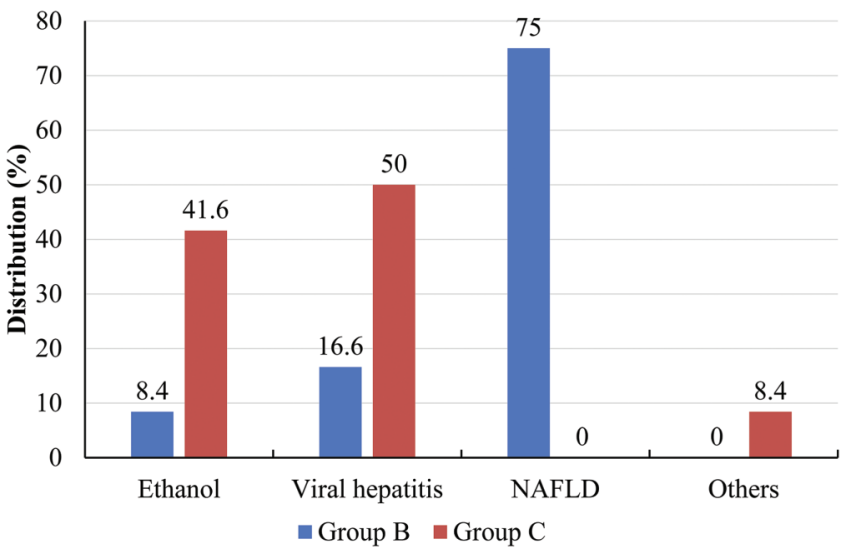

Fig. 1. Etiologic distribution of chronic hepatitis and cirrhosis in Groups B and $\mathrm{C}$ (in\%).

Abbreviation: NAFLD, non-alcoholic fatty liver disease.

were analysed by Binary logistic regression (univariate and multivariate). All statistical tools were two-tailed and level $(p)$ of $<0.05$ was considered as significant. All statistical tests were performed using SPSS for Windows, version 22 (Armonk IBM Corp).

\section{Results}

Seventy-one patients were in Group A, and there were twelve patients in each Group B and Group C. The etiologies of the chronic hepatitis and chronic liver disease cases are shown in Fig. 1 and Supplementary Tables 1 and 2.

\section{Demography and clinical presentation of dengue infection}

As shown in Table 1, males accounted for $61 \%$ (58/95) of the study population and the mean age was significantly higher in Group C (A:39.18 $\pm 1.6, B: 40.83 \pm 16.2$ and C:50.92 \pm 7.90 years; $p=0.02)$. Fever was universally present in all the patients. Jaundice was present in $58.3 \%$ of the patients in Group C, compared to $8.5 \%$ and $8.3 \%$ in Group A and Group B respectively $(p<0.001)$. Similarly, there were a greater number of Group $C$ patients (58.3\%) with ascites, as compared to Group A (7\%) and Group B (none) $(p<0.001)$. Eighty-nine patients had classical DF, two patients presented as DHF, one as ALF and three presented as ACLF. There was 1 each case of DHF in Group A and Group B. Three patients presented as ACLF, including two with HBV as the cause of underlying cirrhosis and one with ethanol-related cirrhosis (Fig. 2 and Supplementary Fig. 1). Dengue serotype 2 was isolated in the samples analyzed.

Table 1. Comparison of demographic features, clinical features and biochemical parameters

\begin{tabular}{|c|c|c|c|c|}
\hline Parameter & $\begin{array}{l}\text { Group A, no liver disease, } \\
n=71\end{array}$ & $\begin{array}{l}\text { Group B, chronic } \\
\text { hepatitis, } n=12\end{array}$ & $\begin{array}{l}\text { Group C, cirrhosis, } \\
n=12\end{array}$ & $p$ \\
\hline Age (years) & $39.18 \pm 1.6$ & $40.83 \pm 16.28$ & $50.92 \pm 7.90$ & 0.02 \\
\hline Male & $39(54.9 \%)$ & $10(83.3 \%)$ & $9(75 \%)$ & 0.1 \\
\hline Jaundice & $6(8.5 \%)$ & $1(8.3 \%)$ & $7(58.3 \%)$ & $<0.001$ \\
\hline $\begin{array}{l}\text { Upper gastrointestinal bleed/ } \\
\text { mucosal bleed }\end{array}$ & $1(1.4 \%)$ & $1(8.3 \%)$ & 0 & 0.26 \\
\hline Altered sensorium & $2(2.8 \%)$ & 0 & 0 & 0.7 \\
\hline Ascites & $5(7 \%)$ & $0(0)$ & $7(58.3 \%)$ & $<0.001$ \\
\hline Gallbladder edema on USG & $27(38 \%)$ & $2(16.7 \%)$ & $6(50 \%)$ & 0.21 \\
\hline Hemoglobin ( $g / d L)$ & $12.88 \pm 2.55$ & $13.56 \pm 1.76$ & $9.23 \pm 1.88$ & $<0.001$ \\
\hline $\operatorname{TLC}\left(\times 10^{3} / \mathrm{mm}^{3}\right)$ & $4.3(1.6-31.9)$ & $3.8(2-8.8)$ & $3.85(2.1-6.6)$ & 0.65 \\
\hline Platelet $\left(\times 10^{3} / \mathrm{mm}^{3}\right)$ & $99(9-354)$ & $80.5(11-281)$ & $65(15-110)$ & 0.02 \\
\hline INR & $1(0.9-6.6)$ & $1(0.8-2)$ & $2(1-7.59)$ & $<0.001$ \\
\hline Creatinine (mg/dL) & $1(0.22-9.25)$ & $0.72(0.15,2)$ & $1(0.01-10)$ & 0.14 \\
\hline Total bilirubin (mg/dL) & $0.96(0.3-27.6)$ & $0.9(0.3,2.6)$ & $5.25(0.48-25.6)$ & 0.002 \\
\hline Direct bilirubin (mg/dL) & $0.2(0.1-19.4)$ & $0.14(0.07-1.6)$ & $2.06(0.06-12.8)$ & 0.002 \\
\hline AST (IU/L) & $129(13-5876)$ & $171(41-539)$ & $38.5(17-111)$ & $<0.001$ \\
\hline ALT (IU/L) & $95(10-4217)$ & $194(63-488)$ & $38(10-177)$ & $<0.001$ \\
\hline SAP (IU/L) & $75(39-500)$ & $98(41-458)$ & $119.5(28-282)$ & 0.09 \\
\hline GGT (IU/L) & $40(8-556)$ & 75 (11-195) & $47.5(15-396)$ & 0.46 \\
\hline Total protein $(\mathrm{g} / \mathrm{dL})$ & $6.12 \pm 0.73$ & $7.16 \pm 0.70$ & $6.91 \pm 0.86$ & 0.005 \\
\hline Albumin (g/dL) & $3.52 \pm 0.77$ & $3.9 \pm 0.42$ & $2.56 \pm 0.54$ & $<0.001$ \\
\hline Globulin (g/dL) & $3.65 \pm 0.9$ & $3.35 \pm 0.58$ & $3.45 \pm 0.63$ & 0.52 \\
\hline Hospital stay (days) & $4.83 \pm 2.88$ & $7.33 \pm 2.3$ & $13 \pm 5$ & $<0.001$ \\
\hline
\end{tabular}

Abbreviations: ALT, alanine transaminase; AST, aspartate transaminase; GGT, gamma-glutamyl transpeptidase; INR, international normalized ratio; TLC, total leukocyte count. 


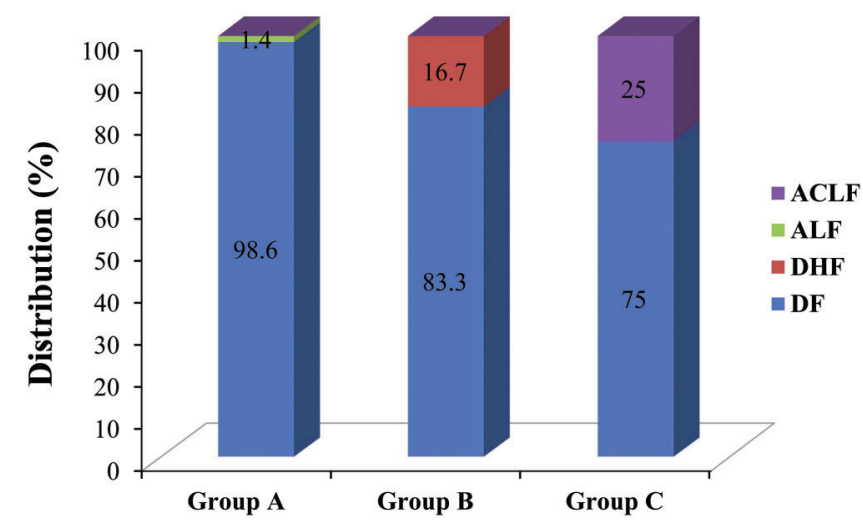

Fig. 2. Distribution of clinical presentation of dengue infection (\%).

Abbreviations: ACLF, acute-on-chronic liver failure; ALF, acute liver failure; DF, dengue fever; DHF, dengue hemorrhagic fever.

\section{Laboratory parameters in the presence of dengue infection}

As shown in Table 1, there was less hemoconcentration in Group C compared to Group A and Group B $(p<0.001)$. Total leukocyte counts were comparable in all the groups. There was non-significant enhanced thrombocytopenia in Group C [platelet values for Group A: 99 (9-354), Group B: 80.5 (11-281), Group C: 65 (15-110); $p=0.27]$. Nonvariceal upper gastrointestinal bleeding was more common in Group A and Group B than in Group C, even though the cirrhotics had significantly prolonged international normalized ratio (INR) and thrombocytopenia.

\section{Liver involvement in normal cases and in chronic liver disease with or without cirrhosis}

As shown in Figs. 3 and 4, the median levels of AST and ALT were significantly low in Group C, probably because of poor hepatic reserve (Table 1 ). Patients in Group $C$ had significantly low albumin compared to those in Group A and Group B. Cirrhotics had significantly prolonged INR [2 (1-7.59)] compared to the patients in Group A [1 (0.9-6.6)] and Group B [1 (0.8$2)](p<0.001)$ attributable to the underlying liver disease.

\section{Clinical course and outcome in normal cases and in chronic liver disease with or without cirrhosis}

As shown in Table 2, 2 patients in Group A had organ failure. The patient presenting as ALF (jaundice to encephalopathy interval of 5 days) had organ failure in the form of renal failure (creatinine $>1.5$ ) and shock (blood pressure $<90 / 60$ on vasopressors), and he recovered. One patient in Group A had DHF with multiorgan failure and ARDS, and succumbed within $48 \mathrm{~h}$. Two patients with ACLF had respiratory failure requiring ventilatory support, and succumbed to pneumonia and superadded sepsis. The third patient in Group $\mathrm{C}$ was discharged against advice and hence was considered as mortality. A total of 3 patients expired in Group $C$ compared to 1 in Group A and none in Group B $(p=0.001)$. Patients in Group C required prolonged hospital stay (in days) compared to those in Group A or Group B (Group A: $4.83 \pm 2.88$, Group B: $7.33 \pm$ 2.3, Group C: $13 \pm 5 ; p<0.001)$.

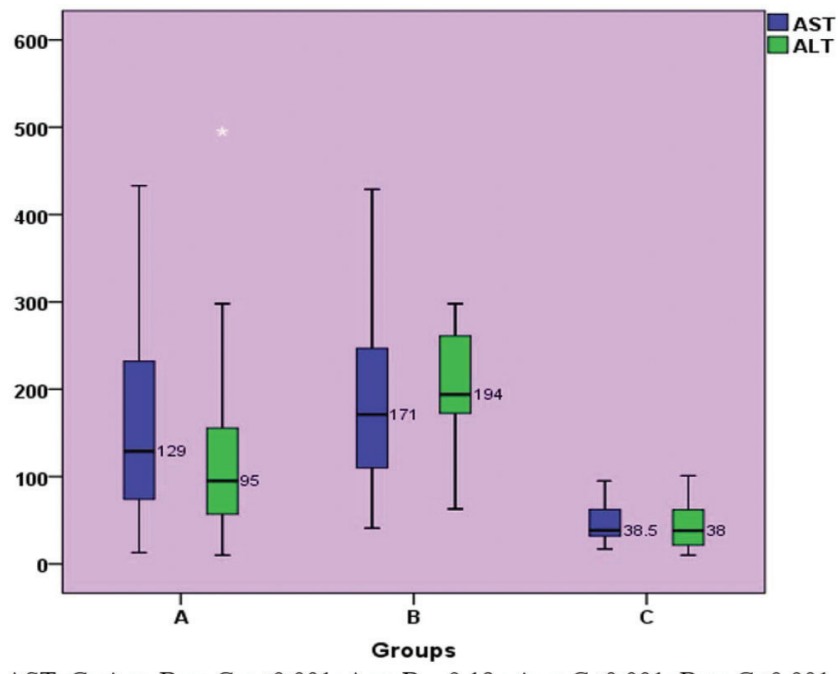

AST- Gr.A vs B vs C-p $<0.001$; A vs B -0.19 ; A vs $\mathrm{C}<0.001$; $\mathrm{B}$ vs $\mathrm{C}<0.001$ ALT- Gr.A vs B vs C-p $<0.001$; A vs B-0.002; A vs C-0.003; B vs $C<0.001$

Fig. 3. Significant difference in AST and ALT in Group C compared to Groups A and B $(p<\mathbf{0 . 0 0 1})$.

Abbreviations: ALT, alanine transaminase; AST, aspartate transaminase.

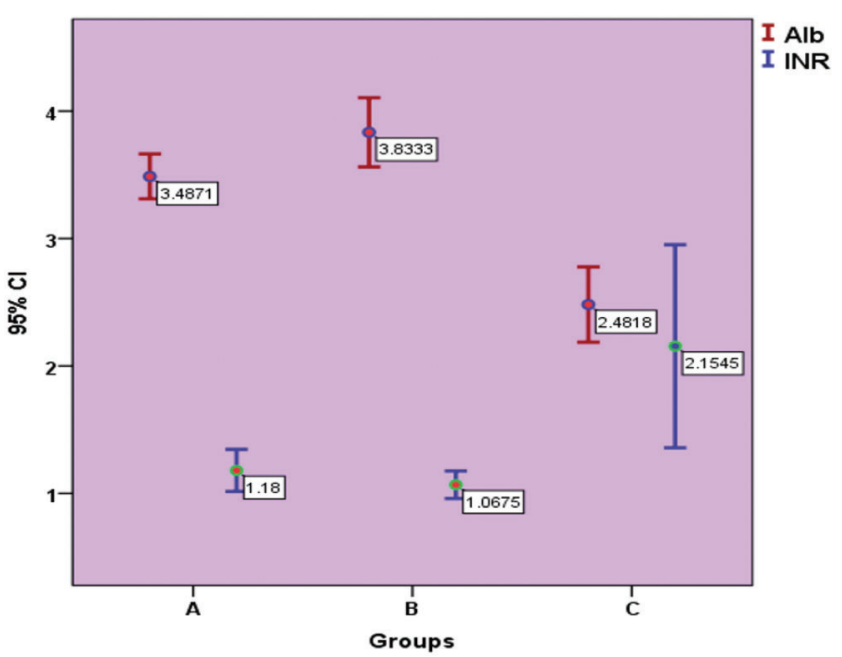

Albumin-Gr.A vs B vs C-p $<0.001$; A vs B-0.13; A vs $\mathrm{C}<0.001$; $\mathrm{B}$ vs $\mathrm{C}<0.001$ INR- Gr.A vs B vs C-p $<0.001$; A vs B-0.62; A vs $\mathrm{C}<0.001$; $\mathrm{B}$ vs $\mathrm{C}<0.001$

Fig. 4. Significant difference in albumin and international normalized ratio in group C (cirrhotics) compared to Groups A and B $(p<0.001)$.

\section{Predictors of liver failure}

As shown in Table 3, on univariate analysis hemoglobin, albumin, INR and bilirubin predicted development of liver failure. On multivariate analysis, it was found that only INR and bilirubin significantly predicted development of liver failure. International normalized ratio (INR) of $>2.03$ (AUROC: 0.97 , sensitivity $100 \%$, specificity $95.7 \%$ ) and bilirubin $>7.05$ (AUROC: 0.97 , sensitivity $100 \%$, specificity $96.7 \%$ ) significantly predicted liver failure in the whole cohort. 
Table 2. Outcome in the study group

\begin{tabular}{lllll}
\hline Outcome & $\begin{array}{l}\text { Without liver disease } \\
(\text { Group A) }\end{array}$ & $\begin{array}{l}\text { With chronic hepatitis } \\
\text { (Group B) }\end{array}$ & $\begin{array}{l}\text { With cirrhosis } \\
\text { (Group C) }\end{array}$ & $p$ \\
\hline Died & $1(1.4 \%)$ & 0 & $3(25 \%)$ & A vs. B vs. C: 0.01 \\
Survived & $70(98.6 \%)$ & $12(100 \%)$ & $9(75 \%)$ & A vs. B: 1 . \\
Total & 71 & 12 & 12 & B vs. C: 0.22 . \\
\hline
\end{tabular}

\section{Discussion}

Dengue infection is the most common arboviral infection in tropical countries. Although literature survey showed many articles on dengue hepatitis, there are none on patients with liver disease and co-infection with dengue virus. To the best of our knowledge, the current study is the first to enumerate the effects of dengue infection in patients with liver disease and to compare thereof with normal individuals infected with dengue.

We found that the dengue infection is common in younger aged people, possibly because of their greater extent of outdoor activities which would render them to be exposed to the causative mosquito. Dengue had significant male predilection, particularly in those with liver disease. The likely explanation for this was inconclusive and the postulation was that the females in India were more covered with dress than their male counterparts, leading to less contact with the mosquito. Patients with cirrhosis had ascites, significantly lower hemoglobin, and platelet count. This difference is attributable to the difference in the synthetic function and associated portal hypertension, with or without hypersplenism. Even though patients had significant thrombocytopenia and prolonged INR, there was no increased risk of mucosal bleeds in the cirrhotic patients.

Deranged liver enzymes are a common phenomenon in individuals with dengue fever and is seen in up to $97 \%$ of cases. ${ }^{5,6}$ Kuo et al. ${ }^{17}$ recruited 270 patients and showed that there is elevation of AST, gamma-glutamyl transpeptidase, ALT, alkaline phosphatase, and bilirubin in $93.3 \%, 83.0 \%$, $82.2 \%, 16.3 \%$, and $7.2 \%$ of the patients, respectively; the study also highlighted that AST elevation is common during the early phase of infection. In our study as well, AST was elevated more than ALT in patients without liver disease, probably suggesting that the source of AST was not only the liver but also the damaged myocytes. ${ }^{18,19}$ There was marginal (less than 2 times ULN) elevation of aminotransferases in cirrhotics compared to patients with chronic hepatitis (commonly up to 4 times ULN) and in patients without any liver disease (mostly around 3times ULN). Kulkarni et al. ${ }^{20}$ in his series of 948 children, showed that there was a raised AST/ALT in $90 \%$ but hyperbilirubinemia was rare, seen only in $0.95 \%$ of cases. There was a marginal increase in the aminotransferases in cirrhotics probably because of reduced synthetic function and reduced viable liver tissue for production of enzymes. Cirrhotics due to sarcopenia may have reduced muscle source of AST as well. Tang et al. ${ }^{21}$ in his case series of 27 patients, showed that HBV and dengue co-infected patients had significantly lower levels of IL- 6 and TNF- $\alpha$ than in patients with only dengue infection, but the actual cause and implication of this was not elucidated.

In another study of 45 confirmed DHF cases, Nguyen et al., ${ }^{22}$ showed that mild to moderate elevation of enzymes (AST >> ALT) was quite common, like in our series, but the elevation did not differ significantly between patients with or without hepatitis $B / C$ infection.

Our study also showed that the serotype of dengue was type 2 in all the cases, irrespective of liver disease, confirming that the most prevalent serotype in India is type 2 . The dengue infection had a varied presentation, as far as liver involvement is concerned (i.e. it presented as anicteric hepatitis, icteric hepatitis, ALF, ACLF and worsening liver failure in a previously decompensated cirrhosis). The incidence of ALF due to dengue infection in adults is reported somewhere between $5.2-8.9 \% .{ }^{10,11}$ Dengue as an etiology for ALF in children varies from $18.5 \%$ in Indian children to as high as $34 \%$ in Thai children. ${ }^{23,24}$ In our adult population, the incidence of ALF was $1.1 \%$ and of ACLF was $3.1 \%$. Dengue leading to acute hepatic decompensation has been rarely reported. ${ }^{12}$ Patients with cirrhosis had a prolonged hospital stay and higher complication rate, leading to significantly higher mortality than in those without cirrhosis or liver disease. Dengue leading to liver failure and subsequent outcome could be predicted independently based upon the presence of jaundice (total serum bilirubin $>7 \mathrm{mg} / \mathrm{dL}$ ) or impaired hepatic synthetic function, i.e. coagulopathy: INR of $>2$.

The study is unique and is the first to report the differences in dengue manifestations in this type of patient population. Deranged LFT in addition to thrombocytopenia and hemoconcentration is a clue for dengue-related liver involvement. Patients with underlying liver disease without cirrhosis can

Table 3. Variables predicting development of liver failure

\begin{tabular}{llr}
\hline & OR $(95 \% \mathrm{CI}, p$ value $)$ & Multivariate \\
\cline { 2 - 3 } Predictors & Univariate & \\
\hline Hemoglobin & $0.65(0.42-1.03,0.07)$ & $2.28(1.14-4.58,0.02)$ \\
Albumin & $0.22(0.04-1.07,0.06)$ & $1.24(1.05-1.46,0.01)$ \\
International normalized ratio & $2.25(1.27-3.97,0.005)$ & $1.24(1.06-1.46,0.007)$ \\
\hline
\end{tabular}


develop liver failure and need to be managed aggressively. For patients with cirrhosis, our study is the first to address the clinical challenge of evaluating pyrexia and identifying dengue. Dengue infection is greatly masked by the liver disease, due to disproportionately low hemoglobin (in contrast to hemoconcentration) and low platelet count, but transient liver enzyme elevation with AST > ALT is a clue for suspecting dengue infection. Jaundice is uncommon with dengue, but the presence of jaundice or coagulopathy could be a red-flag sign. The effects of liver transplant for denguerelated ALF, ACLF or decompensated cirrhosis are largely unknown to date. The current study is the first to highlight the clinical profile, key to early detection as well as to prognosticate this rare cohort of patients.

The limitation of this study is lack of the toxin assay, which could have added more prognostic value. Study of the systemic inflammatory cytokine profile as well as the liver histology could have made our study more robust. We did not find any NASH cirrhotics who developed dengue infection; whether this was due to our time-bound study design or whether NASH is protective, is unknown. The relatively low risk for bleeding manifestation in cirrhosis with dengue infection despite derangement of coagulation profile needs further study. Probably, compensatory mechanisms play a role.

In conclusion, dengue infection is a common cause of fever in Southeast Asia. Patients with underlying cirrhosis may not have classical features of dengue infection but tend to have poor prognosis. Dengue infection can have varied manifestations, ranging from simple fever to ACLF and ALF. Dengue should be suspected as a cause of acute insult, especially in endemic areas. Our results also suggest that a high suspicion for underlying liver disease should be maintained for patients with progressive illness and hepatic dysfunction.

\section{Acknowledgments}

This study had no grant support. This work was presented as a poster at The AASLD Liver Meeting 2016, and AVK had received travel grant award from the Indian Council of Medical research for the same.

\section{Conflict of interest}

The authors have no conflict of interests related to this publication.

\section{Author contributions}

Contributed to study concept and design (AVK, MP, AKC), data acquisition and drafting of manuscript (AVK, MP), microbiological investigations support (EG); data compilation and critical revision (SKS, AKC, AVK), statistical analysis (PJ), important intellectual input, final drafting of the manuscript, administrative and technical support (SKS). All authors approved the final draft.

\section{References}

[1] Bhatt S, Gething PW, Brady OJ, Messina JP, Farlow AW, Moyes CL, et al. The global distribution and burden of dengue. Nature 2013;496:504-507. doi: 10.1038 /nature 12060

[2] World Health Organization. Dengue: Guidelines for diagnosis, treatment, prevention and control (New edition 2009). Available from: https://www. who.int/tdr/publications/documents/dengue-diagnosis.pdf.

[3] Horstick O, Farrar J, Lum L, Martinez E, San Martin JL, Ehrenberg J, et al. Reviewing the development, evidence base, and application of the revised dengue case classification. Pathog Glob Health 2012;106:94-101. doi: 10. 1179/2047773212Y.0000000017.

[4] Souza LJ, Lopes AC, Bastos DA. Icterícia na dengue hemorrágica: relato de três casos. Rev Bras Clin Terap 2002;28:198-201.

[5] Souza LJ, Alves JG, Nogueira RM, Gicovate Neto C, Bastos DA, Siqueira EW, et al. Aminotransferase changes and acute hepatitis in patients with dengue fever: analysis of 1,585 cases. Braz J Infect Dis 2004;8:156-163. doi: 10. 1590/S1413-86702004000200006

[6] Parkash O, Almas A, Jafri SM, Hamid S, Akhtar J, Alishah H. Severity of acute hepatitis and its outcome in patients with dengue fever in a tertiary care hospital Karachi, Pakistan (South Asia). BMC Gastroenterol 2010;10:43. doi: 10.1186/1471-230X-10-43.

[7] Samanta J, Sharma V. Dengue and its effects on liver. World J Clin Cases 2015;3:125-131. doi: 10.12998/wjcc.v3.i2.125.

[8] Nimmannitya S. Clinical spectrum and management of dengue haemorrhagic fever. Southeast Asian J Trop Med Public Health 1987;18:392-397.

[9] Lum LC, Lam SK, George R, Devi S. Fulminant hepatitis in dengue infection. Southeast Asian J Trop Med Public Health 1993;24:467-471.

[10] Deepak NA, Patel ND. Differential diagnosis of acute liver failure in India. Ann Hepatol 2006;5:150-156.

[11] Tan SS, Bujang MA. The clinical features and outcomes of acute liver failure associated with dengue infection in adults: a case series. Braz J Infect Dis 2013;17:164-169. doi: 10.1016/j.bjid.2012.09.007.

[12] Agarwal MP, Giri S, Sharma V, Roy U, Gharsangi K. Dengue causing fulminant hepatitis in a hepatitis B virus carrier. Biosci Trends 2011;5:44-45. doi: 10. 5582/bst.2011.v5.1.44.

[13] Lanciotti RS, Calisher CH, Gubler DJ, Chang GJ, Vorndam AV. Rapid detection and typing of dengue viruses from clinical samples by using reverse transcriptase-polymerase chain reaction. J Clin Microbiol 1992;30:545-551.

[14] Dengue haemorrhagic fever: diagnosis, treatment, prevention and control. 2nd edition. Geneva: World Health Organization. Available from: https: //www.who.int/csr/resources/publications/dengue/Denguepublication/en/.

[15] Sarin SK, Kumar A, Almeida JA, Chawla YK, Fan ST, Garg H, et al. Acute-onchronic liver failure: consensus recommendations of the Asian Pacific Association for the study of the liver (APASL). Hepatol Int 2009;3:269-282. doi: $10.1007 / \mathrm{s} 12072-008-9106-x$.

[16] O'Grady JG, Schalm SW, Williams R. Acute liver failure: redefining the syndromes. Lancet 1993;342:273-275. doi: 10.1016/0140-6736(93)91818-7.

[17] Kuo CH, Tai DI, Chang-Chien CS, Lan CK, Chiou SS, Liaw YF. Liver biochemical tests and dengue fever. Am J Trop Med Hyg 1992;47:265-270. doi: 10 . 4269/ajtmh.1992.47.265.

[18] Nath P, Agrawal DK, Mehrotra RM. Ultrastructural changes in skeletal muscles in dengue virus-infected mice. J Pathol 1982;136:301-305. doi: 10.1002/path.1711360405.

[19] Kalita J, Misra UK, Mahadevan A, Shankar SK. Acute pure motor quadriplegia: is it dengue myositis? Electromyogr Clin Neurophysiol 2005;45:357-361.

[20] Kulkarni MJ, Sarathi V, Bhalla V, Shivpuri D, Acharya U. Clinico-epidemiological profile of children hospitalized with dengue. Indian J Pediatr 2010;77:11031107. doi: 10.1007/s12098-010-0202-2.

[21] Tang Y, Kou Z, Tang X, Zhang F, Yao X, Liu S, et al. Unique impacts of HBV coinfection on clinical and laboratory findings in a recent dengue outbreak in China. Am J Trop Med Hyg 2008;79:154-158. doi: 10.4269/ajtmh.2008.79.154.

[22] Nguyen TL, Nguyen TH, Tieu NT. The impact of dengue haemorrhagic fever on liver function. Res Virol 1997;148:273-277. doi: 10.1016/s0923-2516 (97)88364-1.

[23] Jagadishkumar K, Jain $P$, Manjunath VG, Umesh L. Hepatic involvement in dengue Fever in children. Iran J Pediatr 2012;22:231-236.

[24] Poovorawan Y, Hutagalung Y, Chongsrisawat V, Boudville I, Bock HL. Dengue virus infection: a major cause of acute hepatic failure in Thai children. Ann Trop Paediatr 2006;26:17-23. doi: 10.1179/146532806X90565. 